\title{
Compression for texture images in different basis functions using system criteria analysis
}

\author{
A.Yu. Babikov \\ Southern Federal \\ University, Nekrasovski 44, \\ 347922, Taganrog, \\ Russian Federation; \\ alex12345ander@yandex.ru
}

\author{
V.V. Voronin \\ Don State Technical \\ University \\ Gagarin's square 1 \\ 344000, Rostov-on-Don, \\ Russian Federation \\ voroninslava@gmail.com
}

\author{
V.P. Ryzhov \\ Southern Federal \\ University, \\ Nekrasovski 44, \\ 347922, Taganrog, \\ Russian Federation; \\ vpr_trtu@mail.ru
}

\author{
Yu.V. Ryzhov \\ Southern Federal \\ University, \\ Nekrasovski 44, \\ 347922, Taganrog, \\ Russian Federation; \\ vpr_trtu@mail.ru
}

\begin{abstract}
This paper considers image compression for texture images. For texture representation we consider the orthogonal decomposition of two-dimensional signals (images) using spectral transform in the different basis functions. This paper focuses on the analysis of the following basis DCT, FFT, Haar, Hartley, and Walsh using system criteria analysis. The error of the orthogonal representation of images and the computational cost are considered when choosing a basis system, which is based on the Bellman-Zadeh concept using fuzzy sets. It is shown that the Haar transform can represent textural images more efficiently with smaller average risk than other basis functions.
\end{abstract}

\section{Keywords}

Images, textures, orthogonal bases, fuzzy sets, the Bellman-Zadeh concept

\section{INTRODUCTION}

Two-dimensional signals (images) are the most important class of signals, since a significant part of the documentation, photographs, illustrations to artistic texts, drawings and much more in human activity refers to this class. This class of twodimensional signals occupies a significant (sometimes basic) volume of business and household information. Therefore, despite the significant and ever-increasing possibilities of computer technology, in the foreseeable future the requirement of compress the scope of information of such signals exists and will exist.

One of the most effective types of signal compression is spectral compression algorithms based on the use of systems of orthogonal basis functions (from now on abbreviated as bases). This type of compression is widely described in the literature (for example, in [Sof03]). Nevertheless, many problems have not been resolved to the present.

The problem of a well-founded choice of the orthogonal system of functions is not solved yet because of the many possible selection criteria. Earlier it was shown ([Vor16]) that when solving

Permission to make digital or hard copies of all or part of this work for personal or classroom use is granted without fee provided that copies are not made or distributed for profit or commercial advantage and that copies bear this notice and the full citation on the first page. To copy otherwise, or republish, to post on servers or to redistribute to lists, requires prior specific permission and/or a fee. practical problems in conditions of limited time or other resources, it is expedient to use system criteria, including not only the error of signal representation but also the consumption of computational resources, functional stability, and other system-technical characteristics. Such a systematic approach requires consideration of many aspects of the performance and operation of the system (including interaction with other systems, with the environment) and the use of poorly structured expert information. A significant amount of work is devoted to this problem, but they do not contain specific design techniques at the stage of solving signal problems.

The most important task of primary signal processing is the choice of the form of representation [Zha00]. Many applied problems are solved using the time representation, but the relations in the spectral domain build most of the signals processing algorithms [Buj17]. Most systems require the calculation of convolutional integrals that are most efficiently computed by Fast Fourier Transform algorithms. In this case, the choice of the basis of the spectral expansion is essential. Thus, the number of terms of the series is minimized when using the Karhunen-Loeve basis, but the computational speed is significantly increased, and computational costs are reduced when using the bases of piecewiseconstant functions (Walsh, Haar). The wide application of the basis of trigonometric functions is due to both the physical and familiarity of such a representation, and the most compact solution of subsequent filtration problems. 
The formalized solution of the optimal (in the system-technical sense) choice of basis in [Vor16] is largely due to the use of image models in the form of a random two-dimensional Gaussian field with a given correlation function. Such a model is easily implemented on a computer; it can be generated an almost unlimited number of times with the reproduction of the specified parameters, which is convenient for comparing different processing algorithms. But in many cases the nature of transmitted and processed images is different - these are objects of human activity, urban or natural environment, art objects. Textures are often used as an intermediate variant between abstract computer models and real images [Har79], [Pav16]. Also, in the final analysis, the perception of images is based on the features of the human vision as the ultimate recipient and the ultimate decision-making body. Therefore, the task of jointly examining the objective and subjective characteristics of images and the choice of basic systems, considering these two groups of data, is posed in this research.

\section{PROPOSED SOLUTION}

Let $Z: D \subset R^{2} \rightarrow R^{l}$ be a given source image ( $l=1$ : for a gray-scale image; $l=3$ : for a RGB color image).

We used 4 different class of textures [Har79] (Fig. 1). They are SKI (clouds), WATER (water), STONE (two types of stones).

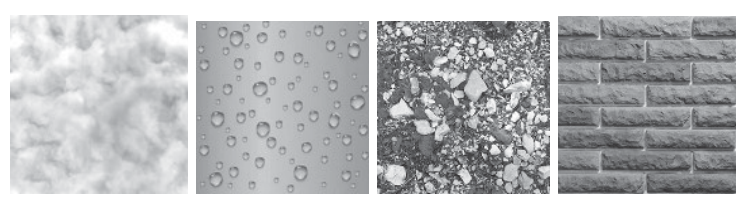

Figure 1. Textures.

For each image, the analysis was performed by the generalized Fourier transform in some basis:

$$
G(n, m)=\frac{1}{N M} \sum_{x=0}^{N-1} \sum_{y=0}^{M-1} Z(x, y) \cdot \varphi_{n m}(x, y) ;
$$

where $N, M$ is the number of counts in coordinates $x, y$, image $Z(x, y), G(n, m)$ - image spectrum in the basis $\varphi_{n m}(x, y)$.

The inverse transform allows you to get the original picture:

$$
Z(x, y)=\sum_{n=0}^{N-1} \sum_{m=0}^{M-1} G(n, m) \cdot \varphi_{n m}(x, y) .
$$

If the number of employed terms of the series (1) is less than the number of samples $(M, N)$, then the compressed image $\widetilde{Z}(x, y)$ differs from the original, calculated by the formula (2). Then the research task was to evaluate the quality of the compressed image.

For each type of texture, the spectrum was calculated in five orthogonal bases: discrete cosine transform (DCT), trigonometric basis (FFT), Haar, Hartley, Walsh. Then the synthesis was performed using the truncated series of images $\widetilde{Z}(x, y)$ that were presented to the experts.

\section{EXPERIMENTAL RESULTS Expert assessments}

A group of 10 experts was organized to obtain expert assessments. Initially, the original images $Z(x, y)$ (each of the four species), and then synthesized $\widetilde{Z}(x, y)$ by truncated series, were presented to the experts. Experts should specify from what number of the removed terms of the series the image can be estimated as excellent, good, satisfactory or unsatisfactory (on a four-point scale).

Nearby the estimate for each basis, the experts had to put the number of terms of the series at which the image remained in the zone of the corresponding estimate. Such criteria as the average value for the number of terms of the series removed and the rootmean-square value of the spread of estimates were determined from the questionnaires of all experts (Table 1). Here, for each image and each basis, the mean values of the bounds of the estimates are given and, through the sign of "/" - the root-mean-square values of the spread of the estimates. The results of calculating the root-mean-square errors associated with the truncation of the series for the textures STONE-1, STONE-2 are shown in Fig. 2, 3.

In many cases of practical importance, it is impossible to obtain all the information from expert practitioners in the form of numbers. Expert opinions can be compactly expressed using the theory of fuzzy sets.

\begin{tabular}{|l|l|l|l|l|}
\hline \multicolumn{1}{|r|}{ Image } & SKI-28 & WATER-2 & STONE-1 & STONE-2 \\
\cline { 1 - 4 } DCT & & & & \\
& $53 / 23$ & $51 / 23$ & $139 / 42$ & $125 / 38$ \\
& $82 / 19$ & $79 / 13$ & $168 / 37$ & $173 / 59$ \\
& $106 / 7$ & $96 / 7$ & $199 / 25$ & $211 / 18$ \\
\hline FFT & $58 / 16$ & $63 / 16$ & $120 / 41$ & $166 / 31$ \\
& $88 / 18$ & $84 / 15$ & $159 / 40$ & $193 / 27$ \\
& $102 / 13$ & $100 / 12$ & $194 / 33$ & $216 / 18$ \\
\hline Haar & $55 / 11$ & $44 / 18$ & $98 / 41$ & $93 / 46$ \\
& $74 / 13$ & $72 / 17$ & $142 / 48$ & $153 / 39$ \\
& $95 / 10$ & $92 / 12$ & $184 / 37$ & $204 / 28$ \\
\hline Hartley & $5 / 3$ & $5 / 2$ & $6 / 3$ & $5 / 3$ \\
& $10 / 11$ & $24 / 21$ & $13 / 5$ & $25 / 31$ \\
& $83 / 42$ & $67 / 35$ & $77 / 57$ & $128 / 85$ \\
\hline Walsh & $37 / 19$ & $51 / 17$ & $80 / 35$ & $92 / 23$ \\
& $72 / 9$ & $75 / 14$ & $142 / 46$ & $149 / 33$ \\
& $97 / 12$ & $98 / 7$ & $200 / 25$ & $202 / 22$ \\
\hline
\end{tabular}

Table 1. Results of the expert evaluation of textures 
The main characteristic of fuzzy sets is the membership function of $\mu_{A}(u)$ an element $u$ to the subset A, which assigns to each element of the set functions can be determined by interviewing the experts (system designers) using known scaling techniques.

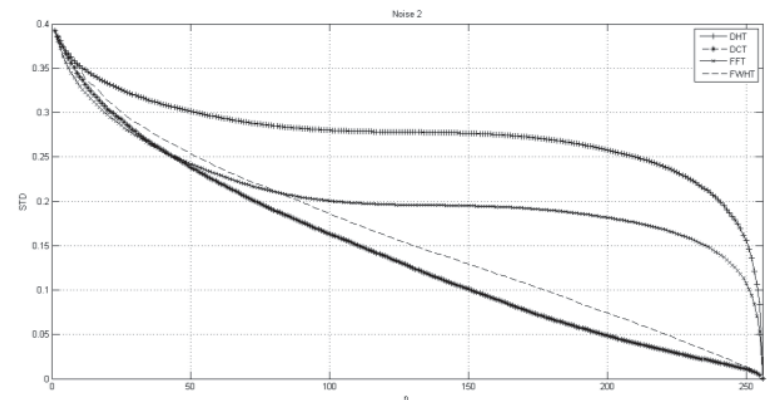

Figure 2. The root-mean-square errors with truncation of the series, Fourier for the STONE-1

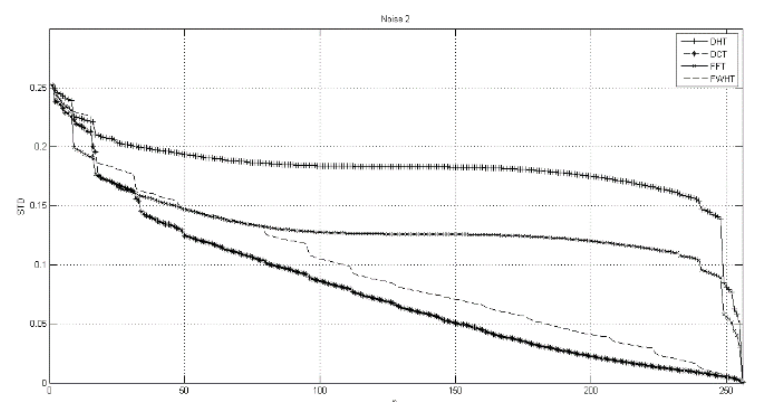

texture.

Figure 3. The root-mean-square errors with truncation of the series, Fourier for the STONE-2 texture.

\section{Calculation system}

The possibilities of achieving the system's objective that is the implementation of the required system characteristics, and the integration of systemtechnical constraints are combined in the BellmanZadeh concept [Bel76], according to it the fuzzy set $D=C \cap H \quad(\mathrm{C}$ is the subset of objectives, $\mathrm{H}$ is the subset of constraints) with the membership function $\mu_{d}(u)$ :

$$
d_{o p t} \in D: \mu_{d}(u)=\mu_{c}(u) \wedge \mu_{h}(u)=\max
$$

It is called a solution, and the optimal value corresponds to the largest value $\mu_{d}(u)$. Thus, the objectives and constraints are merged, which allows optimizing the solution quite simply.

When solving the problem of choosing the spectral description of images, it is possible to use the onedimensional (for square matrices $N \mathrm{x} N$ ) or two- $u \in U$ a number from the interval $[0,1]$ that characterizes the degree of belonging of $u$ to the set $A \subset U$. The membership dimensional (for $N \mathrm{x} M$ matrices) the space of alternatives, which was used as the number of terms of the truncated series $l$.

The membership functions of objectives were defined as the dependence of the image quality on the number of terms used in the Fourier series. It was built by expert assessments. Verbal rates "excellent", "good", "satisfactory", "unsatisfactory" correspond to traditional scores "5", “4”, “3", "2". If you want to increase the significance of any zones of the scale, you can enter non-linear scales or enter the desired non-linear function of the purpose $\mu_{c}(l)$. In our case, using a semilogarithmic scale of estimates, we could use such an approximation of the data in table 2: $\mu_{c}(l)=0,5 \sqrt{l}$ (curve "T" in Fig. 3). The same figure shows the functions of the purpose $\mu_{c}(l)$ and constraints $\mu_{h}(l)$, constructed according to the data of Table. 2 for the SKY-28 image using the FFT basis. In Fig. 4 shows similar membership functions but using the basis of Haar functions.

To introduce the constraint membership function $\mu_{h}(l)$, we used the number of computational operations (equivalent additions) when computing a given array of spectral components for different bases, these data are given in Table. 2 (from [Vor13]).

\begin{tabular}{|l|l|}
\hline The type of basis & $\begin{array}{l}\text { The number of } \\
\text { equivalent series } \\
\text { (image size } N \times N \text { ) }\end{array}$ \\
\hline $\begin{array}{l}\text { Fourier } \\
\text { transformation }\end{array}$ & $24 N^{2} \log _{2} N$ \\
\hline Cosine transform & $10 N^{2} \log _{2} N$ \\
\hline Hartley transform & $12 N^{2} \log _{2} N+5 N^{2}$ \\
\hline Walsh transform & $2 N^{2} \log _{2} N$ \\
\hline Haar transformation & $4 N^{2}-2$ \\
\hline
\end{tabular}

Table 2. Number of computational operations of algorithms of two-dimensional spectral transformations

In Table 3 the values of the number of computational operations for bases of trigonometric functions and Haar functions are given as an example.

Because modern computers and microprocessors have achieved high performance, it is possible to smooth out the dependence $\mu_{h}(l)$ using nonlinear transformations, for example: 


$$
\mu_{h}(u)=\frac{4}{4+\lg \frac{S(l)}{S(16)}},
$$

where $S(l)$ is the number of computational operations for $l$ terms of the Fourier series.

\begin{tabular}{|l|l|l|l|l|}
\hline Basis/N & 16 & 32 & 64 & 128 \\
\hline FFT & $2,46 \cdot 10^{4}$ & $1,23 \cdot 10^{5}$ & $5,88 \cdot 10^{5}$ & $1,74 \cdot 10^{6}$ \\
\hline Haar & $1,02 \cdot 10^{3}$ & $4,09 \cdot 10^{3}$ & $1,64 \cdot 10^{4}$ & $6,55 \cdot 10^{4}$ \\
\hline
\end{tabular}

Table 3. The number of operations in the calculation of spectra in bases of trigonometric functions and Haar functions

The membership of constraints $\mu_{h}(l)$ using (4) is also shown in Fig. 4 and 5 . The abscissa of the intersection point $\mu_{c}(l)$ and by the Bellman-Zadeh concept allows determining the optimal number of series terms $l_{\text {ost }}$, which is indicated in the figures.

\section{SKY-28, FFT}

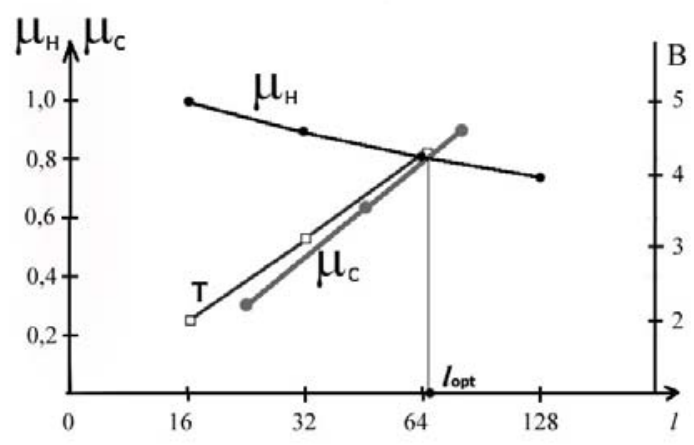

Figure 4. The membership functions of the objective $\mu_{c}(l)$ and constraints $\mu_{h}(l)$ for the basis of trigonometric functions.

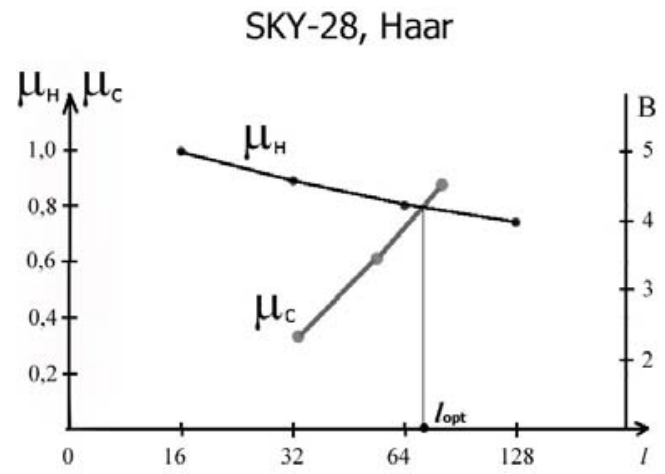

Figure 5. The membership functions of the objective $\mu_{c}(l)$ and constraints $\mu_{h}(l)$ for the basis of Haar functions.

\section{CONCLUSION}

Although the optimal values of the number of series terms turned out to be very close for bases of trigonometric functions and Haar functions, in the
Haar basis the number of calculations is less by one or two orders of magnitude, which determines its advantage in using signal detection and recognition problems. However, for tasks related to signal filtering, it is preferable to use the basis of trigonometric functions.

In general, although the expert estimates are correlated with the mean-square error, more acceptable for practice, especially when evaluating objects familiar to humans. When choosing the best basic system, we should consider both the error in the orthogonal representation of images and the laboriousness of the calculations, which is quite simply carried out by the Bellman-Zadeh concept using fuzzy sets.

\section{ACKNOWLEDGMENTS}

This work was supported by Russian Ministry of Education and Science in accordance to the Government Decree № 218 from April 9, 2010 (project number № 074-11-2018-013 from May 31, 2018 (03.G25.31.0284)).

\section{REFERENCES}

[Bel70] Bellman R.E., Zadeh L.A. Decision-Making in Fuzzy Environment // Management Science, vol. 17, pp.141- 160, 1970.

[Buj17] Bujack R., Flusser J. Flexible Moment Invariant Bases for 2D Scalar and Vector Fields. International Conference in Central Europe on Computer Graphics, Visualization and Computer Vision WSCG, pp. 11-20, 2017.

[Har79] Haralick R. Statistical and structural approaches to texture. Proceedings of the IEEE, 67, pp. 786-804, 1979.

[Pav16] Pavie N., Gilet G., Dischler J.-M., Ghazanfarpour D. Procedural Texture Synthesis by Locally Controlled SpotNoise. International Conference in Central Europe on Computer Graphics, Visualization and Computer Vision WSCG, pp. 71-80, 2016.

[Sof03] Soifer V.A. Methods of computer image processing. Fizmatlit Publ, pp. 784, 2003.

[Vor16] Voronin V.V., Ryzhov V.P., Marchuk V.I., Makov S.V. Texture representations in different basis functions for image synthesis using system criteria analysis. IS\&T D International Symposium on Electronic Imaging, Image Processing: Algorithms and Systems XIV 2016, pp. 1-6(6), 2016.

[Zha00] Zhang J., Fieguth P., and Wang D. Random field models. In A. Bovik, editor, Handbook of Image and Video Processing, Academic Press, San Diego, pp. 301-312, 2000. 